\title{
Pain assessment according to the International Spinal Cord Injury Pain classification in patients with spinal cord injury referred to a multidisciplinary pain center
}

\author{
S Mahnig ${ }^{1}$, G Landmann ${ }^{2}$, L Stockinger ${ }^{2}$ and E Opsommer ${ }^{1}$
}

Study design: This is a retrospective study.

Objectives: The aim of this study was to investigate the epidemiology of pain types in patients with spinal cord injury (SCI) according to the International Spinal Cord Injury Pain (ISCIP) classification.

Setting: This study was conducted in a multidisciplinary pain center.

Methods: Socio-demographic and clinical data were examined and ISCIP classification was applied.

Results: Sixty-six individuals ( $51 \pm 13$ years) with $\mathrm{SCl}$ had pain, a lesion older than 5 years in $67 \%$ and a pain history older than 5 years in $54 \%$ of patients. According to the ISCIP classification, nociceptive pain was present in $58 \%$ (musculoskeletal pain) and $3 \%$ (visceral pain) of the patients. At-level, below-level neuropathic pain and other neuropathic pain were observed, respectively in 53 , 42 and $5 \%$ of patients. Unknown pain type was found in $8 \%$ of patients. Patients with complete lesions showed significantly more frequent neuropathic pain $(P=0.021)$ and more frequent at-level SCI pain $(P=0.00)$ compared with those with incomplete lesions. Patients with paraplegia had more often at-level pain $(P=0.00)$, whereas patients with tetraplegia reported more often below-level pain $(P=0.00)$. Patients had severe pain (mean intensity: $8.2( \pm 1.6)$ on a 0 to 10 numerical scale) and showed high grades of pain chronicity. Mild to severe depression and anxiety were present, respectively in 53 and $56 \%$ of patients. The health-related quality of life was low.

Conclusion: The use of the ISCIP classification in a clinical setting is mirroring the very complex pain situation in patients with SCI referred to a multidisciplinary pain center, and it might be an important step for adequate pain therapy.

Spinal Cord (2016) 54, 809-815; doi:10.1038/sc.2015.219; published online 12 January 2016

\section{INTRODUCTION}

Pain is one of the most prevalent secondary conditions after spinal cord injury (SCI), which leads to reduced quality of life and poorer rehabilitation outcomes. ${ }^{1,2}$ Around half to two-thirds of all people with SCI have pain. ${ }^{3}$ The SCI pain management is challenging and the outcomes are unsatisfactory. ${ }^{4}$ Difficulties in diagnosis and classification of SCI pain led to the need for a clear classification of different pain types, which was implemented by an international group of SCI pain specialists (researchers and clinicians) and reviewed by international SCI organizations, such as American Spinal Injury Association (ASIA) and the International Spinal Cord Society (ISCoS). The newly developed International Spinal Cord Injury Pain (ISCIP) classification was designed to be a comprehensive, simple and acceptable protocol, and to replace all existing classifications. ${ }^{5}$ It is mechanism-based and distinguishes between nociceptive pain, neuropathic pain, other pain and unknown pain. Nociceptive pain is defined as 'pain arising from activation of nociceptors', where a nociceptor is defined as 'a sensory receptor that is capable of transducing and encoding noxious stimuli'. ${ }^{6}$ Subtypes of nociceptive pain are musculoskeletal pain, visceral pain and other nociceptive pain. ${ }^{5}$ Neuropathic pain is defined as 'pain caused by a lesion or disease of the somatosensory nervous system'. ${ }^{7}$ In SCI, it is subdivided into at-level SCI (neuropathic) pain, below-level SCI (neuropathic) pain and other (neuropathic) pain, whereas the latter is unrelated to SCI. ${ }^{5}$ At-level SCI pain is the neuropathic pain caused by spinal cord or nerve root damage that occurs at the neurological level of injury (NLI) and/or within three dermatomes below NLI but not in any lower dermatomes. ${ }^{5}$ Pain that is thought to be caused by cauda equina damage is also classified as at-level pain. ${ }^{5}$ Below-level SCI pain is perceived more than three levels below NLI, and it is thought to be caused by a lesion or disease affecting the spinal cord. ${ }^{5}$ Pain is classified as 'other pain' if there is no detectable noxious stimulus, inflammation or damage to the nervous system responsible for the pain. ${ }^{7}$ If a type of pain cannot be clearly assigned to any of the before-mentioned categories, it will be classified as 'unknown pain'. The classification provides detailed information regarding pain characteristics to differentiate pain types and subtypes. The reliability of use of the ISCIP classification by clinicians using a clinical vignette approach was found to be moderate, and neuropathic pain was challenging to classify. ${ }^{8}$

\footnotetext{
${ }^{1}$ University of Health Sciences (HESAV), University of Applied Sciences and Arts Western Switzerland (HES-SO), Lausanne, Switzerland and ${ }^{2}$ Centre for Pain Medicine, Swiss Paraplegic Centre, Nottwil, Switzerland

Correspondence: Dr E Opsommer, University of Health Sciences (HESAV), University of Applied Sciences and Arts Western Switzerland (HES-SO), Av. de Beaumont, 21, $\mathrm{CH}-1011$ Lausanne, Switzerland.

E-mail: Emmanuelle.opsommer@hesav.ch

Received 19 May 2015; revised 21 October 2015; accepted 11 November 2015; published online 12 January 2016
} 
It seems to be unclear whether there are associations between pain and demographic or medical variables in individuals with SCI in contrast to the general chronic pain population. ${ }^{9}$ Previous research in SCI found no association between pain locations or ratings of pain intensity and demographic or medical variables. ${ }^{2,10}$ In several studies, they found no clear differences in prevalence of pain for gender and SCI characteristics. ${ }^{3,10-12}$ A prospective cohort study found that people with tetraplegia were more likely to report below-level neuropathic pain than people with paraplegia. ${ }^{13}$ One study found a higher pain prevalence in patients older than 60 years compared with younger patients; ${ }^{14}$ another study found patients with below-level SCI pain to be significantly younger than those without below-level SCI pain. ${ }^{12}$ In addition, a positive correlation between pain prevalence and depression, as well as for depression and higher levels of pain, seems to exist in $\mathrm{SCI}^{3}$ The socioeconomic circumstances may also have an important role. Indeed, financial hardship has been shown to be consistently associated with more secondary conditions, comorbidities and pain, whereas mental health, participation and quality of life were reduced. ${ }^{15}$

Since the ISCIP classification and its initial validation using vignettes were published in 2012, only one study could be found that applied it to SCI patients during the first year after injury. ${ }^{12}$ Therefore, the objective of this study was to classify a retrospective data set of patients with SCI and chronic pain who were referred to a multidisciplinary pain center according to the ISCIP. In addition, associations between SCI pain types and demographic or disease-related variables were explored.

\section{MATERIALS AND METHODS}

\section{Setting and subjects}

For this retrospective study, data from patients of a multidisciplinary pain center in Switzerland were analyzed. Data of all patients with SCI treated from January 2011 to December 2013 have been collected in a standardized form. Inclusion criterion was an injury of the spinal cord related to trauma or to degenerative spinal disease. Patients younger than 18 years, patients without pain, patients with other neurological disorders (for example, multiple sclerosis, Guillain-Barre syndrome, acute disseminated encephalomyelitis), congenital conditions, tumor, vascular diseases of spinal cord and patients with incomplete data were excluded from the analysis. According to the national Human Research Ordinance, there is no need for an ethical request for irrevocably anonymized retrospective studies. ${ }^{16}$

\section{Clinical data collection}

Demographic data included basic personal data (age, gender) and general questions regarding SCI (cause of injury, time since injury and reason for referral), as well as medication on admission to the pain center. The assessment of neurological function after SCI was performed by a neurologist according to the International Standards for Neurological Classification of Spinal Cord Injury (ISNCSCI). ${ }^{17}$ These recommendations include the neurological status with determination of NLI and the extent of injury on the ASIA Impairment scale (AIS grade). Diagnosis of SCI was confirmed by spinal imaging techniques, and the site of lesion (myelon, cauda equina or both) was defined. Pain-related data such as pain character and the maximum intensity of each pain type were assessed. The pain intensity was defined on a 0-10 Numerical Rating Scale (NRS), with $0=$ 'no pain' and $10=$ 'worst possible pain'. According to the detailed pain history, the complete neurological examination including ASIA score and pain drawings done by the patient showing all pain sites, each pain was matched separately by a neurologist with the pain characteristics given by the classification. Thus, each pain could be classified according to the three tiers of the ISCIP classification: ${ }^{5,8}$

Tier 1: Pain type (nociceptive pain, neuropathic pain, other pain or unknown pain).
Tier 2: Pain subtype (musculoskeletal pain, visceral pain, other nociceptive pain, at-level SCI pain, below-level SCI pain or other neuropathic pain).

Tier 3: Primary pain source and/or pathology.

Patients-reported outcomes were collected by the standardized German pain questionnaire, designed by the German Association for the Study of Pain (DGSS), as time since occurrence of pain, number of pain sites, pain-related disability, psychological status and general health. ${ }^{18}$ The psychological status was assessed by the use of the Hospital Anxiety and Depression Scale (HADS). ${ }^{19}$ This widely used questionnaire consists of 14 items with possible scorings from $0-3$ in order to assess levels of anxiety (seven items) and depression (seven items). The cutoff for a positive test is $8+$ on each subscale..$^{19,20}$ Adequate to excellent validity of this questionnaire in SCI people has been demonstrated. ${ }^{21,22}$ Health-related quality of life was determined by the SF-12 questionnaire. ${ }^{23}$ The SF-12 questionnaire, a shortened version of the widely used SF-36, describes the degree of general physical health (physical component summary) and mental health (mental component summary). ${ }^{23}$ It has been validated for SCI people. ${ }^{24}$ Chronic pain severity was assessed using the Graded Chronic Pain Scale (GCPS), which consists of seven questions related to pain intensity (three items), pain-related disability (three items) and the number of disability days (one item). ${ }^{25} \mathrm{~A}$ score for pain intensity is calculated by the average of actual pain, worst pain and average pain, whereas the disability score represents a quantified mean value of impairment in daily activities, social activities and work activities. Both scores are assessed on a 0 to 100 scale. From these parameters, grades of chronic pain severity (0-IV) can be identified. ${ }^{25}$ The disability subscale has been validated for people with SCI. $^{26}$ The grade of chronification of pain was defined by the Mainz Pain Staging System (MPSS). ${ }^{27}$ This is a questionnaire consisting of 11 items to assign the pain into one of three possible stages of chronification. It showed good construct and prognostic validity in patients with different pathologies. ${ }^{28,29}$

\section{Statistical analyses}

Data analysis was carried out using SPSS software (IBM SPSS Statistics for Windows, Version 21.0. IBM, Armonk, NY, USA). A descriptive analysis was performed on the demographic, clinical and SCI characteristics. Data are reported as means and standard deviations. $t$-tests and Mann-Whitney $U$-tests were used for continuous variables; $\chi^{2}$ and Fisher's exact test were used for binary variables. To calculate correlations between characteristics, Pearson's correlation coefficient $(r)$ or Spearman's rank correlation $\left(r_{\mathrm{s}}\right)$ was used depending on the data level. $P$-values $<0.05$ were considered to be statistically significant.

\section{RESULTS}

\section{Demographic characteristics}

During the period of January 2011 to December 2013, 86 individuals with neurologic spinal disorders were referred and treated at the multidisciplinary pain center. Nineteen patients were excluded from analysis because of other neurologic disorders (multiple sclerosis $(n=6)$, Guillain-Barre syndrome $(n=3)$, spinal ischemia $(n=3)$, tumor $(n=2)$, spastic spinal paralysis $(n=2)$, lipomeningomyelocele $(n=1)$, myelitis $(n=1)$, acute disseminated encephalomyelitis $(n=1))$, and one patient was excluded because of missing values. Finally, we analyzed data of 66 patients ( 17 women and 49 men) with a mean age of 51 years $( \pm 13)$ who had SCI and pain. The lesion was older than 5 years in $67 \%$ of patients, whereas $55 \%$ had a pain history of more than 5 years. The pain was severe, with a mean intensity of $8.2( \pm 1.6)$ on NRS. Almost three quarter of the patients reported pain in the legs or feet, and more than half of them had pain in the pelvic region. One-third of the patients had back pain and almost the same number in the ventral upper body. Most often reported pain sites for nociceptive pain were pelvic region (24\%), back (23\%), leg/foot (17\%) and shoulder (14\%), whereas neuropathic pain sites were most frequently in the lower extremity (62\%). Among the cohort, two patients had severe visceral pain. The clinical characteristics of the included patients are summarized in Tables 1 and 2 . 
Table 1 Clinical characteristics of patients $(n=66)$

\begin{tabular}{lc}
\hline Variable & $\mathrm{n}(\%)$ \\
\hline Gender & \\
Female & $17(26 \%)$ \\
Male & $49(74 \%)$ \\
& \\
Age (years) & \\
Mean ( \pm s.d.) & $51.3( \pm 13.2)$ \\
Median; range & $50.5 ; 19-78$ \\
& \\
Age at injury (years) & \\
Mean ( \pm s.d.) & $38.7( \pm 17.0)$ \\
Median; range & $36.5 ; 13-75$ \\
AlS grade & \\
A & \\
B & $27(41 \%)$ \\
C & $6(9 \%)$ \\
D & $8(12 \%)$ \\
E & $25(38 \%)$ \\
\end{tabular}

$\begin{array}{ll}\begin{array}{l}\text { Classification } \\ \text { Paraplegia }\end{array} & 40(61 \%) \\ \text { Tetraplegia } & 26(39 \%) \\ & \\ \text { Lesion site } & \\ \text { Myelon } & 38(58 \%) \\ \text { Myelon and cauda equina } & 13(20 \%) \\ \text { Cauda equina only } & 15(23 \%)\end{array}$

Time since injury (years)

Mean $( \pm$ s.d.)

Median; range

$12( \pm 11.9)$

8.5; $0-44$

$$
\begin{aligned}
& \text { Pain since } \\
& <1 \text { year } \\
& >1 \text { year } \\
& >2 \text { years } \\
& >5 \text { years } \\
& >10 \text { years }
\end{aligned}
$$

$13(20 \%)$

$8(12 \%)$

$9(14 \%)$

$11(17 \%)$

$25(38 \%)$

Number of pain subtypes per patient

1

2

3 or more

$30(45 \%)$

$26(39 \%)$

$10(15 \%)$

Abbreviation: AIS, American Spinal Injury Association Impairment Scale.

\section{ISCIP classification and SCI characteristics}

According to the ISCIP classification, nociceptive pain was present in $61 \%$, neuropathic pain in $79 \%$ and unknown pain in $8 \%$ of the patients. The neuropathic pain (both at-level and below-level) was the most common pain type regardless of the type of the lesion (myelon, cauda equina or both) or the AIS grade. The distribution of pain types and subtypes according to the ISCIP classification is illustrated in Figure 1a. It shows that the musculoskeletal pain was the most prevalent of pain subtypes (58\%), followed by at-level (53\%) and below-level (42\%) neuropathic pain. More than half of our patients showed more than one pain subtype (Table 1). As shown in Figure 1b, illustrating the distribution of pain subtypes within lesion types, at-level SCI (neuropathic) pain was proportionally more frequent in patients with cauda equina lesion than in patients with myelon lesion,
Table 2 Lesion site and AIS grade

\begin{tabular}{lccc}
\hline & Myelon lesion & Cauda equina lesion & Myelon and cauda equina lesion \\
\hline AIS A & 14 & 6 & 7 \\
AIS B & 5 & 0 & 1 \\
AIS C & 5 & 2 & 1 \\
AIS D & 14 & 7 & 4 \\
\hline
\end{tabular}

Abbreviation: AIS, American Spinal Injury Association Impairment Scale.

as neuropathic pain in cauda equina is always defined as at-level pain. The distribution of pain subtypes within each AIS grade is also presented in Figure 1c. At-level SCI (neuropathic) pain was most often reported in patients with complete lesions (AIS A), whereas patients with AIS D lesion reported more frequently musculoskeletal pain.

As reported in Table 3, patients with a complete lesion showed significantly more frequent neuropathic pain compared with those with incomplete lesions ( 93 vs $69 \% ; P=0.02$ ), and more frequent at-level SCI pain $(74 \%$ vs $38 \% ; P=0.00)$ compared with those with an incomplete lesion. For below-level SCI pain, there was no frequency difference between complete (44\%) and incomplete (41\%) lesions. Patients with paraplegia reported more often at-level pain $(73 \%)$ in comparison with people with tetraplegia $(23 \% ; P=0.00)$. Patients with tetraplegia had significantly more often below-level pain than patients with paraplegia ( 65 vs $28 \% ; P=0.00$ ). There were no significant differences between socio-demographic (age, gender) or SCI characteristics and pain subtypes.

Pain intensity, pain descriptors and pharmacological treatment Nociceptive pain was reported with an intensity of NRS $7.4( \pm 1.8)$, whereas the neuropathic pain was rated slightly higher $(8.1 \pm 1.8)$ but not significantly $(P=0.09)$. There were no differences between at-level and below-level pain intensity. The reported maximum pain intensity for each pain subtype is shown in Table 4. Patients with nociceptive pain described their pain predominately as pinprick (40\%), pressurelike $(25 \%)$ and pulling (23\%); patients with neuropathic pain had mainly burning (79\%), shooting (35\%) and electric shock-like (25\%) pain, while multiple descriptors were possible. On admission to the multidisciplinary pain center, the average number of medications was $2.6( \pm 1.5$; range: $0-7)$ per patient. Patients had calcium-channel antiepileptics (61\%), analgesics (38\%), opioids (32\%), antidepressants such as mirtazapine and trazodone (26\%), baclofen (24\%), benzodiazepines $23 \%$, serotonin norepinephrine reuptake inhibitors (SNRI; 15\%), tricyclic antidepressants (TCA; 12\%) sodium-channel antiepileptics $(6 \%)$. Patients with neuropathic pain only $(35 \%)$ had calcium-channel antiepileptics (61\%), opioids (39\%), benzodiazepines $(35 \%)$, analgesics $(26 \%)$, antidepressants such as mirtazapine and trazodone $(26 \%)$, SRNI (22\%), TCA (17\%) and baclofen (13\%).

\section{Patient-reported outcomes}

Outcomes of 43 individuals who completed the German pain questionnaire are reported in Table 5. A total of 23 incomplete questionnaires or questionnaires that were not filled in were excluded. Mild to severe anxiety and depression assessed by the HADS were present in more than half of the patients, in 56\% and 53\%, respectively. The health-related quality of life assessed by the SF-12 Health Survey was low (mean physical component summary: $29.3 \pm 9.4$; mean mental component summary: 42.9 \pm 12.3 ). According to the outcome of GCPS, nearly two-thirds of patients had the highest grade of chronic pain severity (Grade IV). MPSS questionnaire showed the highest stage of pain chronification for half 


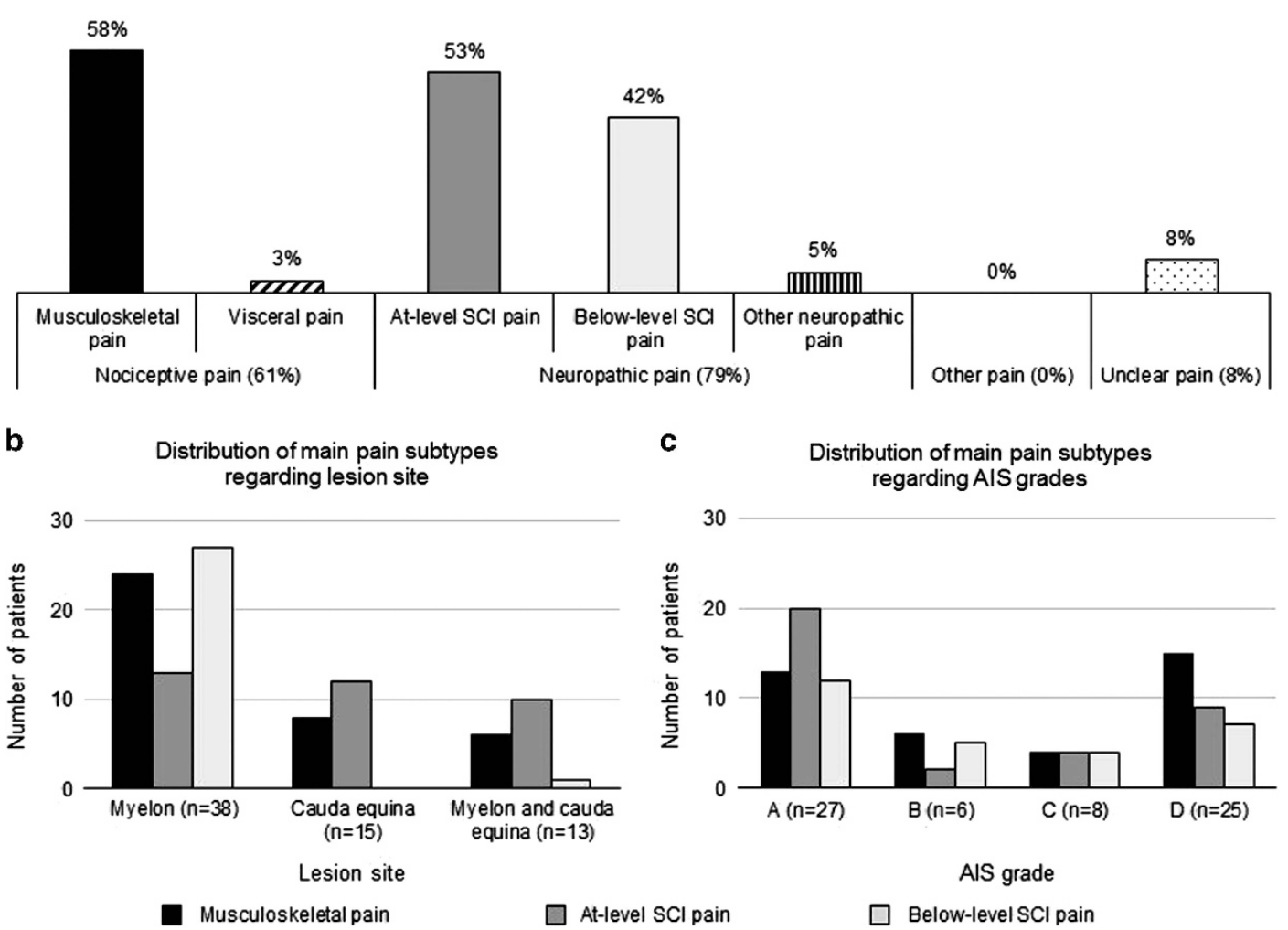

Figure 1 Pain subtypes according to the International Spinal Cord Injury Pain (ISCIP) classification in a cohort of individuals with spinal cord injury referred to a multidisciplinary pain center (a). Distribution of main pain subtypes regarding the lesion site (b) and the AIS (American Spinal Injury Association Impairment Scale) grade (c).

Table 3 Distribution of pain types in complete/incomplete lesions and paraplegia/tetraplegia

\begin{tabular}{lccc}
\hline Pain type & Complete lesion $(\mathrm{n}=27)$ & Incomplete lesion $(\mathrm{n}=39)$ & P-value $\left(\chi^{2}\right)$ \\
\hline Nociceptive pain & $56 \%$ & $64 \%$ & 0.49 \\
Neuropathic pain & $93 \%$ & $69 \%$ & $0.02^{*}$ \\
At-level SCl pain & $74 \%$ & $38 \%$ & $0.00^{*}$ \\
Below-level SCl pain & $44 \%$ & $41 \%$ & 0.78 \\
Other neuropathic pain & $11 \%$ & $0 \%$ & 0.06 (Fisher's exact test) \\
Unclear pain & $4 \%$ & $10 \%$ & 0.31 (Fisher's exact test) \\
\hline Pain type & Paraplegia (n=40) & Tetraplegia (n=26) \\
\hline Nociceptive pain & $60 \%$ & $62 \%$ & \\
Neuropathic pain & $83 \%$ & $73 \%$ & \\
At-level SCl pain & $73 \%$ & $23 \%$ & 0.90 \\
Below-level SCl pain & $28 \%$ & $65 \%$ & 0.36 \\
Other neuropathic pain & $8 \%$ & $0 \%$ & $0.00^{*}$ \\
Unclear pain & $8 \%$ & $8 \%$ & $0.00^{*}$ \\
\hline
\end{tabular}

Abbreviation: SCl, spinal cord injury.

$* \mathrm{P}<0.05$.

of the patients. No significant differences between pain subtypes and the outcomes of the questionnaires could be detected. Similarly, no associations could be found between outcomes of the questionnaires and age, time since injury and time since onset of pain.

Nevertheless, a positive correlation was found between higher levels of depression (HADS-D) and higher pain intensity assessed by GCPS pain scale $\left(r_{\mathrm{s}}=0.33, P<0.05\right)$. In addition, there was an association between high pain intensity (GCPS) and low mental health (SF-12 mental component summary; $r_{\mathrm{s}}=-0.33 ; P=0.03$ ) but no association between pain intensity and physical health (SF-12 physical component summary; $r_{\mathrm{s}}=-0.01 ; P=0.94$ ). The grade of chronification (MPSS) had no significant association with pain intensity $\left(r_{s}=0.17 ; P=0.28\right)$. Except that patients with incomplete injury reported higher levels of anxiety (HADS-A) than patients with 
Table 4 Pain intensity for each pain subtype according to the ISCIP classification

\begin{tabular}{|c|c|c|c|c|c|c|}
\hline \multirow{2}{*}{$\begin{array}{l}\text { Pain type (tier 1) } \\
\text { Pain subtype (tier 2) }\end{array}$} & \multicolumn{2}{|c|}{ Nociceptive pain } & \multicolumn{3}{|c|}{ Neuropathic pain } & \multirow[t]{2}{*}{ Unknown pain } \\
\hline & Musculo-skeletal pain & Visceral pain & At-level SCl pain & Below-level SCI pain & Other neuropathic pain & \\
\hline \multicolumn{7}{|l|}{ Maximum NRS } \\
\hline Mean ( \pm s.d.) & $7.3( \pm 1.8)$ & $9.0( \pm 1.4)$ & $8.0( \pm 2.0)$ & $8.0( \pm 1.5)$ & $8.0( \pm 0.0)$ & $7.6( \pm 2.3)$ \\
\hline Pain sites $(n)$ & 35 & 2 & 34 & 27 & 2 & 5 \\
\hline
\end{tabular}

Abbreviations: ISCIP, International Spinal Cord Injury Pain classification; NRS, numerical rating scale.

Table 5 Outcomes of the self-administered questionnaires

\begin{tabular}{|c|c|}
\hline Assessment & Result (mean \pm s.d.; range; $\mathrm{n}=43$ \\
\hline Pain intensity (maximum NRS) & $8.2( \pm 1.6 ; 1-10)$ \\
\hline \multicolumn{2}{|l|}{ HADS } \\
\hline Anxiety & $8.6( \pm 4.7 ; 0-18)$ \\
\hline Depression & $8.2( \pm 5.5 ; 1-19)$ \\
\hline \multicolumn{2}{|l|}{$S F-12$} \\
\hline PCS & $29.3( \pm 9.4 ; 17.7-50.7)$ \\
\hline MCS & $42.9( \pm 12.3 ; 21.3-66.7)$ \\
\hline \multicolumn{2}{|l|}{ GCPS } \\
\hline Grade 0-1 & $n=2(5 \%)$ \\
\hline Grade 2 & $n=5(12 \%)$ \\
\hline Grade 3 & $n=9(21 \%)$ \\
\hline Grade 4 & $n=27(63 \%)$ \\
\hline \multicolumn{2}{|l|}{ MPSS } \\
\hline Stage I & $n=4(9 \%)$ \\
\hline Stage II & $n=17(40 \%)$ \\
\hline Stage III & $n=22(51 \%)$ \\
\hline
\end{tabular}

Abbreviations: GCPS, graded chronic pain scale; HADS, hospital anxiety and depression scale; MCS, mental component summary; MPSS, mainz pain staging system; NRS, numerical rating scale; PCS, physical component summary; SF-12, short-form SF-12 health survey.

complete lesion $(10.1( \pm 5.1)$ vs $6.2(\mathrm{c} 3.1), P=0.01)$, subgroup analysis showed no significant differences between groups in the outcomes of the questionnaires for gender, paraplegia vs tetraplegia, neuropathic pain vs no neuropathic pain and for all pain subtypes.

\section{DISCUSSION}

The objective of this study was to classify a retrospective data set of patients with SCI and chronic pain who were referred to a multidisciplinary pain center according to the ISCIP classification. In addition, associations between SCI pain types and demographic or disease-related variables were explored.

\section{Usefulness of the ISCIP classification}

From the authors' clinical perspective using the patients neurological and pain history, clinical neurological examination, ASIA scoring and patients pain drawing, it was possible to define pain types and subtypes in most of the reported pain sites within the first visit, even in complex chronic patients with long-term disability and pain following SCI. Only $8 \%$ of the patients showed pain types that remained unknown. This means that for the very minority of the patients, further diagnostic workup was necessary. Therefore, in our opinion, the application of the ISCIP classification is easy to use and with regard to the high rate of classifiable pain types within the first visit. This may help provide appropriate available treatment options immediately, and it may save costs for extensive differential workup or wrong treatment. In comparison with the previous available pain classification for SCI pain of the International Association for the study of Pain (IASP) ${ }^{30}$, the ISCIP classification has the advantage of classifying any type of pain related or unrelated to SCI. In a multidisciplinary pain center it is an advantage to address all pain issues using one classification. For instance, the change from 'above level neuropathic pain' to 'other neuropathic pain' gives the possibility to code neuropathic pain conditions below the injury level, which are unrelated to SCI such as polyneuropathy or additional traumatic nerve lesions. In addition, the possibility to code distinct non-neuropathic pain syndromes such as 'fibromyalgia' under 'other pain' is an advantage. We assume that the thorough use of the items of the ISCIP classification will lead to a more patient-tailored long-term management of their pain condition. Although the reliability of use of the ISCIP classification by minimal trained clinicians using a clinical vignette approach is reported as moderate ${ }^{8}$, at that stage in our experience the use of the classification in clinical setting can be encountered.

\section{Socio-demographic data}

Regarding mean age, our cohort is comparable with the literature of chronic pain patients ${ }^{31,32}$ and similar ${ }^{12}$ or older ${ }^{13}$ in comparison to other SCI cohorts. Basically, more women are affected in the chronic pain population, ${ }^{31,32}$ which is not reflecting our patient group. The sex distribution of our cohort $(74 \% \mathrm{men})$ is similar to the general SCI population in Switzerland $(72 \% \mathrm{men}) .{ }^{15} \mathrm{In}$ contrast, slightly more men were affected in other SCI cohorts $\left(83 \%,{ }^{13} ; 88 \%,{ }^{12}\right)$.

\section{Pain-related data}

The severity of pain problems is suspected to be higher in our population in comparison with people with SCI. Indeed, data from a population-based community survey of the Swiss Spinal Cord Injury Cohort Study (SwiSCI) with a traumatic or nontraumatic SCI reported severe pain ( $>6$ on NRS) in less than $30 \%$ of the participants. ${ }^{15}$ In an SCI cohort, 58\% reported severe and excruciating pain at 5 years following SCI. ${ }^{13}$ In comparison, severe pain was reported by all the patients except three in our sample (95\%), with a mean pain intensity of NRS 8.2. Hence, it can be concluded that only patients who are no longer able to manage their pain with their general practitioner are referred to the multidisciplinary pain center. The distribution of highest pain intensity for visceral pain, followed by similar pain intensities for at-level and below-level SCI pain and lower intensity for musculoskeletal pain in our cohort, is similar to those reported previously. ${ }^{13}$

\section{Pain chronicity, pain severity, psychic comorbidity and quality} of life

In our SCI cohort, the prevalence of higher stadiums of pain chronicity according to MPSS is comparable to those reported in 
chronic noncancer pain. ${ }^{32}$ With regarding to the relationship between pain intensity and interference with activities examined by GCPS, our patient population was worse than in previous reported studies of patients with SCI pain. In our group, two-thirds of the patients showed the highest grade of pain severity in comparison with $16.4 \% .^{13}$ More in accordance to our results, $45 \%$ of patients with uncontrolled non-SCI neuropathic pain noted that their disease was severely or extremely interfering with their daily activities. ${ }^{31}$

According to HADS, our cohort showed signs of anxiety and depression, which is in concordance with chronic noncancer pain patients. ${ }^{33}$ In addition, in patients with uncontrolled neuropathic pain in a pain clinic, depression was reported in $53 \%$ and anxiety in $43 \%,{ }^{31}$ comparable to our cohort (53 and 56\%). A worse mood assessment was found in patients with SCI pain in comparison with those without pain. ${ }^{13}$

Ratings for health-related quality of life was low for physical and mental issues, which is similar to chronic pain patients without SCI. ${ }^{32}$ Similarly, $50 \%$ of patients with uncontrolled neuropathic pain rated their overall health as bad or very bad. ${ }^{31}$

In accordance with a previous review, a significant correlation was found between higher levels of depression (HADS-D) and higher pain intensity assessed by GCPS pain scale. ${ }^{3}$ In addition, there was an association between pain intensity (GCPS) and mental health (SF-12 mental subscale) but no association between pain intensity and physical health (SF-12 physical subscale). Indeed, pain has a broad impact on the physical, cognitive, emotional and social functioning of individuals with SCI. ${ }^{34,35}$ Faced with the difficulties of pain treatments, the pain is a source of great psychological distress among people with SCI. ${ }^{36}$

Hence, mainly patients with a high impact of pain, high levels of depression/anxiety, low level of QOL and a high chronification were treated at the pain center. In summary, our patients with SCI pain show similar or even worse psychosocial parameters than patients with chronic noncancer pain and uncontrolled neuropathic pain. ${ }^{31,32}$

\section{Pain types}

More than half of our patients reported more than one pain type. Multiple pain sites were found in one-third of chronic pain patients, ${ }^{37}$ which is lower than in our population. The presence of two or more pain problems usually of different pain types is frequent for individuals with SCI. ${ }^{38}$

A recent study found at-level pain in 54\%, below-level pain in $66 \%$ and both at-level and below-level pain in $20 \%$ of patients with SCI. ${ }^{39}$ Contradictory to our study, below-level pain was reported more often than at-level pain, which may be explained by varying definition of pain types. One study could be found that reported results according to the ISCIP classification of 90 SCI patients within the first year after injury. ${ }^{12}$ They found an overall pain prevalence of around $80 \%$. As in our study, musculoskeletal pain was the most common pain type, followed by at-level SCI pain and below-level SCI pain, whereas the prevalence of visceral pain was between 0 and 3\%. Although it is not possible to directly compare our results to prevalence studies, the distribution of pain types and subtypes was in accordance with previous research. ${ }^{12,13}$ In contrast to previous studies, ${ }^{12}$ we found some relations between injury characteristics and pain types. Patients with complete lesions reported significantly more often neuropathic at-level pain compared with those with incomplete lesions. Patients with paraplegia had more often at-level pain, whereas patients with tetraplegia reported more often below-level pain; the latter aspect has already been described. ${ }^{13}$ In accordance with previous research pain intensity was not related to injury characteristics or demographic variables. $^{2,10}$

\section{Pain descriptors}

The descriptors of pain quality were more or less in accordance with previous studies, although different questionnaires were used. ${ }^{12,39}$ In our study, both at-level pain and below-level pain were reported as burning (60 and 71\%), but shooting almost exclusively occurred in at-level pain (43 vs 7\%). Another study found hot-burning (60\%), stabbing (58\%) and shooting (55\%) as descriptors for neuropathic pain but without a difference between at-level and below-level for shooting pain. ${ }^{12}$

\section{Current treatment}

At entry, the patients reported the medication they received to treat their pain before consulting the pain center. They mainly reported analgesics for nociceptive pain and antiepileptics for neuropathic pain. Indeed, the pharmacological management of neuropathic pain stays in the mainstream. Yet, the guidelines for the pharmacological management of neuropathic pain recommend first-line medications (for example gabapentin/pregabalin-antiepileptics, tricyclic antidepressants or topical lidocaine) as initial treatment and second-line drug (tramadol or high potent opioids). ${ }^{40,41}$ Nevertheless, analgesics were also prescribed for patients with neuropathic pain only $(26 \%)$. It appears that there are some discrepancies between practice and guidelines, which may be an expression of general difficulties in the pain management after SCI. Because of the retrospective design of our study, more detailed information regarding previous medication and other types of current or previous treatments such as physiotherapy, interventional or psychological therapy were not available. Therefore, an evaluation of previous treatments and in what way the application of the ISCIP classification might have changed the treatment and its efficacy could not be performed.

The present study has some limitations. The sample size could be insufficient to detect significant values for subgroup analyses. In addition, patients had often more than one pain type, making a comparison between pain types difficult. Furthermore, because of the study's retrospective design, there was less control over variables, some data were missing and there is a potential information bias.

\section{CONCLUSION}

The application of the ISCIP classification in the clinical setting is mirroring the very complex pain situation in patients with SCI consulting a multidisciplinary pain center and might be an important step for adequate pain therapy. More specific multimodal therapy strategies according to ISCIP pain types need to be further investigated with longitudinal prospective cohort studies.

\section{DATA ARCHIVING}

There were no data to deposit.

\section{CONFLICT OF INTEREST}

The authors declare no conflict of interest.

1 Jensen MP, Chodroff MJ, Dworkin RH. The impact of neuropathic pain on health-related quality of life: review and implications. Neurology 2007; 68: 1178-1182.

2 Ullrich PM, Jensen MP, Loeser JD, Cardenas DD. Pain intensity, pain interference and characteristics of spinal cord injury. Spinal Cord 2008; 46: 451-455.

3 van Gorp S, Kessels AG, Joosten EA, van Kleef M, Patijn J. Pain prevalence and its determinants after spinal cord injury: a systematic review. Eur J Pain 2014; 19: 5-24. 
4 Finnerup NB, Baastrup C. Spinal cord injury pain: mechanisms and management. Cur Pain Headache Rep 2012; 16: 207-216.

5 Bryce TN, Biering-Sorensen F, Finnerup NB, Cardenas DD, Defrin R, Lundeberg T et al. International spinal cord injury pain classification: part I. Background and description. Spinal Cord 2012; 50: 413-417.

6 Loeser JD, Treede RD. The Kyoto protocol of IASP basic pain terminology. Pain 2008; 137: 473-477.

7 Treede RD, Jensen TS, Campbell JN, Cruccu G, Dostrovsky JO, Griffin JW et al. Neuropathic pain: redefinition and a grading system for clinical and research purposes. Neurology 2008; 70: 1630-1635.

8 Bryce TN, Biering-Sorensen F, Finnerup NB, Cardenas DD, Defrin R, Ivan E et al. International Spinal Cord Injury Pain (ISCIP) classification: Part 2. Initial validation using vignettes. Spinal Cord 2012; 50: 404-412.

9 Breivik H, Collett B, Ventafridda V, Cohen R, Gallacher D. Survey of chronic pain in Europe: prevalence, impact on daily life, and treatment. Eur J Pain 2006; 10 287-333.

10 Nakipoglu-Yuzer GF, Atci N, Ozgirgin N. Neuropathic pain in spinal cord injury. Pain Physician 2013; 16: 259-264.

11 Dijkers M, Bryce T, Zanca J. Prevalence of chronic pain after traumatic spinal cord injury: a systematic review. J Rehabil Res Dev 2009; 46: 13-29.

12 Finnerup NB, Norrbrink C, Trok K, Piehl F, Johannesen IL, Sorensen JC et al. Phenotypes and predictors of pain following traumatic spinal cord injury: a prospective study. J Pain 2014; 15: 40-48.

13 Siddall PJ, McClelland JM, Rutkowski SB, Cousins MJ. A longitudinal study of the prevalence and characteristics of pain in the first 5 years following spinal cord injury. Pain 2003; 103: 249-257.

14 Guzelkucuk U, Demir Y, Kesikburun S, Yasar E, Yilmaz B. Spinal cord injury in older population in Turkey. Spinal Cord 2014; 17: 103.

15 Fekete C, Siegrist J, Reinhardt JD, Brinkhof MW. Is financial hardship associated with reduced health in disability? The case of spinal cord injury in Switzerland. PLOS ONE 2014; 9: e90130.

16 The Federal Authorities of the Swiss Confederation. Ordinance on Human Research with the Exception of Clinical Trials of 20 September 2013 (=HRO; RS 810.301). Available at http://www.admin.ch/opc/en/classified-compilation/20121177/index.html 2014.

17 Kirshblum SC, Burns SP, Biering-Sorensen F, Donovan W, Graves DE, Jha A et al. International standards for neurological classification of spinal cord injury (revised 2011). J Spinal Cord Med 2011; 34: 535-546.

18 Nagel B, Gerbershagen HU, Lindena G, Pfingsten M. Development and evaluation of the multidimensional German pain questionnaire. Schmerz 2002; 16: 263-270.

19 Zigmond AS, Snaith RP. The hospital anxiety and depression scale. Acta Psychiatr Scand 1983; 67: 361-370.

20 Bjelland I, Dahl AA, Haug TT, Neckelmann D. The validity of the Hospital Anxiety and Depression scale. An updated literature review. J Psychosom Res 2002; 52 69-77.

21 Muller R, Cieza A, Geyh S. Rasch analysis of the Hospital Anxiety and Depression Scale in spinal cord injury. Rehabil Psychol 2012; 57: 214-223.

22 Sakakibara BM, Miller WC, Orenczuk SG, Wolfe DL. A systematic review of depression and anxiety measures used with individuals with spinal cord injury. Spinal Cord 2009; 47: 841-851.
23 Ware J Jr, Kosinski M, Keller SD. A 12-Item Short-Form Health Survey: construction of scales and preliminary tests of reliability and validity. Med Care 1996; 34: 220-233.

24 Wilson JR, Hashimoto RE, Dettori JR, Fehlings MG. Spinal cord injury and quality of life: a systematic review of outcome measures. Evid Based Spine Care J 2011; 2: 37-44.

25 Von Korff M, Ormel J, Keefe FJ, Dworkin SF. Grading the severity of chronic pain. Pain 1992; 50: 133-149.

26 Raichle KA, Osborne TL, Jensen MP, Cardenas D. The reliability and validity of pain interference measures in persons with spinal cord injury. J Pain 2006; 7: 179-186.

27 Gerbershagen U. Organized treatment of pain. Determination of status. Der Internist 1986; 27: 459-469.

28 Huppe M, Maier C, Gockel H, Zenz M, Frettloh J. Success of treatment in higher stages of pain chronification as well? An evaluation of the Mainz pain staging system based on the QUAST-analysis sample. Schmerz 2011; 25: 77-88.

29 Frettloh J, Maier C, Gockel H, Huppe M. Validation of the German Mainz Pain Staging System in different pain syndromes. Schmerz 2003; 17: 240-251.

30 Siddall P, Yezierski RP, Loeser J. Pain following spinal cord injury: clinical features, prevalence and taxonomy. IASP Newsletter 2000, 3: 3-7.

31 de Andres J, de la Calle JL, Perez M, Lopez V. Clinical characteristics, patient-reported outcomes, and previous therapeutic management of patients with uncontrolled neuropathic pain referred to pain clinics. Pain Res Treat 2014; 2014: 518716.

32 Frettloh J, Maier C, Gockel H, Zenz M, Huppe M. Characterization of chronic pain patients in German pain centers: core data from more than 10000 patients. Schmerz 2009; 23: 576-591.

33 Manchikanti L, Singh V, Datta S, Cohen SP, Hirsch JAAmerican Society of Interventional Pain Physicians. and Comprehensive review of epidemiology, scope, and impact of spinal pain. Pain Physician 2009; 12: E35-E70.

34 Norrbrink C, Lofgren M, Hunter JP, Ellis J. Patients' perspectives on pain. Top Spinal Cord Inj Rehabil 2012; 18: 50-56.

35 Jensen MP, Moore MR, Bockow TB, Ehde DM, Engel JM. Psychosocial factors and adjustment to chronic pain in persons with physical disabilities: a systematic review. Arch Phys Med Rehabil 2011; 92: 146-160.

36 Putzke JD, Richards JS, Hicken BL, DeVivo MJ. Interference due to pain following spinal cord injury: important predictors and impact on quality of life. Pain 2002; 100: 231-242.

37 Davies HT, Crombie IK, WA M. Where does it hurt? Describing the body locations of chronic pain. Eur J Pain 1998; 2: 69-80.

38 Bryce T. Pain management in persons with spinal cord injury. In: Lin VW (ed.). Spinal Cord Medicine. Principles and Practice 2nd ed. Demos Medical: New York, NY, USA, 2010.

39 Jang JY, Lee SH, Kim M, Ryu JS. Characteristics of neuropathic pain in patients with spinal cord injury. Ann Rehabil Med 2014; 38: 327-334.

40 Attal N, Cruccu G, Baron R, Haanpaa M, Hansson P, Jensen TS et al. EFNS guidelines on the pharmacological treatment of neuropathic pain: 2010 revision. Eur J Neurol 2010; 17: 1113-1123, e67-e88.

41 Dworkin RH, O'Connor AB, Audette J, Baron R, Gourlay GK, Haanpaa ML et al. Recommendations for the pharmacological management of neuropathic pain: an overview and literature update. Mayo Clin Proc 2010; 85: S3-S14. 